
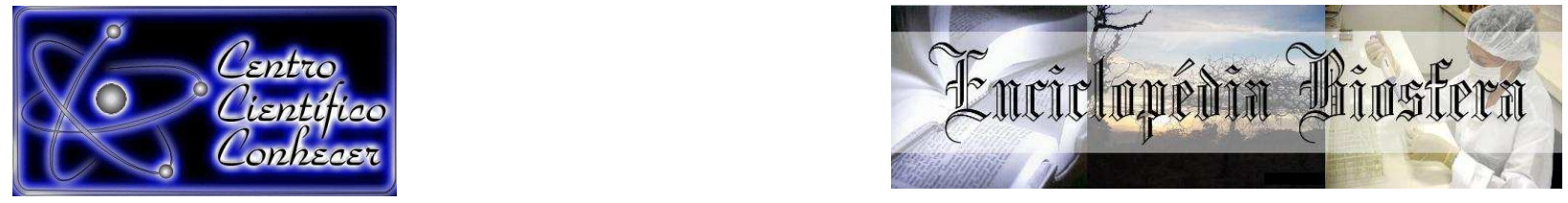

\title{
USO DE CAL NA ESTABILIZAÇÃO GRANULOMÉTRICA E QUÍMICA DE SOLO DE ESTRADAS FLORESTAIS
}

\author{
Breno Santos Arrivabeni ${ }^{1}$; Carlos Cardoso Machado ${ }^{2}$; Giovani Levi Sant'Anna ${ }^{3}$ \\ ${ }^{1}$ Mestrando em Ciências Florestais. Universidade Federal de Viçosa. Viçosa, Minas \\ Gerais, Brasil. breno.arrivabeni@hotmail.com \\ ${ }^{2}$ Professor titular do Departamento de Engenharia Florestal. Universidade Federal \\ de Viçosa. Viçosa, Minas Gerais, Brasil \\ ${ }^{3}$ Pós-doutor em Ciência Florestal. Universidade Federal de Viçosa. Viçosa, Minas \\ Gerais, Brasil
}

\section{Recebido em: 03/10/2016 - Aprovado em: 21/11/2016 - Publicado em: 05/12/2016 DOI: 10.18677/EnciBio 2016B 100}

RESUMO
O presente estudo teve por objetivo analisar o uso de cal como estabilizante granulométrico e químico em um solo de cascalho utilizado para estradas florestais. As amostras coletadas foram submetidas à padronização granulométrica apresentada pelo DNIT, isto é, segundo especificação por faixas. Trabalhou-se com o solo em sua constituição natural e nas faixas supracitadas, sendo que a estas foi acrescido cal no teor de $8 \%$ em relação à massa de solo seco. Inicialmente caracterizou-se o cascalho e posteriormente procedeu-se ao ensaio CBR (Índice de Suporte Califórnia). Em seguida, realizou-se 0 ensaio RCS (Resistência à Compressão Simples) através da confecção de corpos de provas e utilizando-se de dados oriundos da compactação do material. Tais ensaios seguiram as exigências da norma DNER-ME 180/94, a qual estipula para cura de 14 dias o valor de 2,17 Mpa. As análises CBR evidenciaram resultados superiores a 100\%, e aquelas referentes a RCS, estabilizadas com cal e curadas por 14 dias, apresentaram resultados abaixo do valor de referência. Os valores abaixo do estipulado na norma podem, possivelmente, ser solucionados modificando-se a energia empregada, de intermediária para modificada. Tal mudança seria mais adequada quando comparada à alternativa de aumento dos teores de cal, devido a questões econômicas. Os materiais tratados com cal apresentaram, de forma geral, alta capacidade de suporte e de resistência, implicando em um bom comportamento quanto à estabilidade e frente à perda de material por erosão e pelo tráfego.

PALAVRAS-CHAVE: Estabilidade do solo. Índice de suporte Califórnia. Resistência à compressão simples.

\section{LIME USE IN THE CHEMICAL AND GRANULOMETRICAL STABILIZATION OF SOIL FOR FOREST ROADS}

\begin{abstract}
This work aimed to analize the chemical and granulometric stabilization of a gravel soil for forest roads through the use of lime. The sample was analized according to DNIT's granulometric range, adding lime to each layer, representing $8 \%$ of the dry soil mass, and also its natural granulometric characteristics. The development of this
\end{abstract}


analysis was based on the gravel characterization followed by the compaction tests and the California Bearing Ratio (CBR). From the results obtained in the compaction, the "proof bodies" were made to be tested for Resistance to Simple Compression (RSC). The tests were made based on the standard provided by DNER-ME 180/94, which suggests a $2.17 \mathrm{MPa}$ value to a 14 day curing time. The results found from the CBR analysis are above $100 \%$. For the RSC tests that were stabilized and cured for 14 days, the results were below standards. The change of the applied energy, from intermediate to modified, might be a more suitable solution to this issue compared to the increase in the amount of lime, due to its cost. Therefore, these materials, when mixed with lime, present high support and resistance capacity, which indicates good parameters related to stability and erosion, meaning smaller amounts of the material would be lost due to water precipitation or vehicle trafficking.

KEYWORDS: Soil Stability. California Bearing Ratio. Resistance to Simple Compression.

\section{INTRODUÇÃO}

Diante da importância da rede viária nacional e da necessidade de tráfego em qualidade, a conservação de estradas torna-se algo imprescindível e de atenção constante. Em se tratando de estradas rurais e florestais, essa atenção deve ser diferenciada visto que são estas as mais sujeitas à perda de material por intemperismo, tráfego e instabilidade, e as quais são maioria em nosso país. Segundo o SISTEMA NACIONAL DE VIAÇÂO (2016) e a CONFEDERAÇÃO NACIONAL DO TRANSPORTE (2016), o Brasil apresenta $1.720 .643 \mathrm{~km}$ de rodovias, porém com apenas $210.618 \mathrm{~km}$ pavimentados, correspondendo a $12,2 \%$ da malha rodoviária nacional.

O uso de intervenções no solo a fim de garantir a estabilidade dos agregados tem papel fundamental na dinâmica rodoviária. Nesse sentido, a estabilização de determinado solo implica na adição de fatores estabilizantes junto ao mesmo, no intuito de modificar suas propriedades físicas e químicas e proporcionar melhoria ao material. Segundo GONDIM (2008), esses fatores de estabilização podem ser de natureza química, granular ou mesmo energia dinâmica aplicada.

A estabilização mecânica é aquela cujos procedimentos e técnicas mudam o arranjo entre as partículas do solo ou sua granulometria, tais como a compactação, a qual implica na expulsão de ar dos espaços vazios, conferindo ao solo uma nova organização em sua estrutura (GONDIM, 2008).

A estabilização química baseia-se na adição de um material químico (seja cimentante ou não) junto ao solo, atribuindo a este melhorias em suas características. Para atuar como estabilizante químico de um solo modificando suas propriedades físicas e químicas, o material, ao ser adicionado, reage como agente impermeabilizante, dispersante, floculante ou agregante dos materiais presentes no solo (SILVA, 1968).

Ao abordarem os estabilizantes químicos, LAMBE \& MICHAELS (1954) apontaram mecanismos essenciais para que estes modifiquem as propriedades físicas do solo. Dentre tais mecanismos, citam a natureza da formação das partículas hidrofóbicas; o aumento da repulsão interpartículas e a atuação das moléculas-pontes entre partículas, facilitando a coesão interparticular.

A estabilização granulométrica consiste em se obter um material de estabilidade maior que os solos de origem e de porcentagem limitada de partículas finas, com a mistura íntima homogeneizada de dois ou mais solos e sua posterior 
compactação (VIZCARRA, 2010). A identificação dos solos conforme as suas propriedades de textura, plasticidade e comportamento é o mecanismo pelo qual o Sistema Unificado de Classificação dos Solos (SUCS) os reúne. Esse sistema leva em consideração a porcentagem de pedregulhos, areias e finos; a forma da curva granulométrica; a plasticidade e a compressibilidade (SOUSA, 2013).

A cal viva, também conhecida como cal virgem, geralmente se apresenta na forma de grãos, e seus tamanhos podem variar dependendo do processo de fabricação a que foi submetida (OLIVEIRA, 2011). Segundo o DICIONÁRIO LIVRE DE GEOCIÊNCIAS (2016), a produção da cal dá-se pelo processo conhecido como calcinação, que consiste na extração e moagem do calcário e sua submissão a altos fornos industriais, resultando no óxido de cálcio.

A estabilização solo-cal é uma técnica empregada na área de pavimentação que visa principalmente a melhoria permanente das características dos solos, obtendo um aumento na resistência à ação da água, em seu poder de suporte e na trabalhabilidade de solos argilosos. E quando comparada ao emprego de cimento Portland, cimento asfáltico ou materiais mais nobres, a estabilização de solos finos através do uso de cal pode ser uma alternativa de custo relativamente baixo (KLINSKY et al., 2012). Porém, GUYER (2011) defende o uso e as vantagens do cimento Portland como agente cimentante, evidenciando assim alternativas químicas além da cal para estabilização de pavimentos.

No Brasil, a área de estabilização de solos vem se mostrando bastante promissora para a utilização da cal em inúmeros tipos de aplicações, com destaque para a construção de sub-bases e bases rodoviárias (FERRAZ, 1994 e PEREIRA, 2005 citados por OLIVEIRA et al., 2009). Além disso, segundo ARAUJO et al. (2016), a cal além de atuar como estabilizante de solos em camadas granulares, pode atuar também como fíler em misturas asfálticas como melhorador da adesividade.

Segundo ALCÂNTARA (1995) e PEREIRA (2005), citados por OLIVEIRA et al., (2009), as alterações que ocorrem nos solos após a adição de cal se devem a reações de troca catiônica, de carbonatação e, a mais importante, pozolânicas. A ocorrência da reação pozolânica, que é a reação entre a sílica e a alumina do solo com a cal, resulta na formação de vários tipos de agentes cimentantes que são considerados como a maior causa do aumento de resistência mecânica percebida nas misturas solo-cal. MUKESH \& PATEL (2012) afirmam que essas reações pozolânicas são as mesmas que conferem resistência quando da utilização de cimento. Ainda, segundo KLINSKY et al. (2014) comprova, a adição de cal hidratada a solos reativos melhora substancialmente a resistência dos mesmos.

As recomendações de empresas tais como MINASCAL (2016), a saber, defendem a cal hidratada como um excelente reagente, o qual favorece a estabilidade e a impermeabilidade, apresentando ótima relação custo $\mathrm{x}$ benefício. Dessa forma, o objetivo deste estudo foi avaliar a estabilização de solo de estradas florestais para diferentes granulometrias através do uso de cal, analisando-se os efeitos desse aditivo químico nas propriedades físicas e mecânicas do solo. Como objetivo específico destaca-se a escolha da combinação granulométrica de solo que apresente maior estabilidade, resistência e, consequentemente, melhores resultados frente à erosão.

\section{MATERIAL E MÉTODOS}

O solo trabalhado foi coletado numa cascalheira presente na cidade de Cajuri, Minas Gerais, a qual fornece materiais para o revestimento primário das estradas 
florestais da região. Para o procedimento das análises, utilizou-se a cal comercial como produto estabilizante. Fez-se uso de peneiras específicas com diferentes aberturas de malhas, e quando necessário, empregou-se frações de brita 1 e de brita 0 a fim de adequar o material estudado às faixas granulométricas $A, C$ e $F$ propostas pelo DNIT (2006).

A Tabela 1 apresenta a granulometria (\% em peso retido) para cada faixa granulométrica, conforme adaptação da porcentagem em peso passante do DNIT (2006). Destaca-se em especial as faixas de interesse do estudo: A, C e F, a saber.

TABELA 1. Base granular em porcentagem de peso retido por peneiras, para cada faixa de interesse.

\begin{tabular}{|c|c|c|c|c|c|c|c|}
\hline \multicolumn{2}{|c|}{ PENEIRA } & \multirow{2}{*}{ A } & \multirow{2}{*}{ B } & \multirow{2}{*}{ C } & \multirow{2}{*}{ D } & \multirow{2}{*}{$E$} & \multirow{2}{*}{$\mathbf{F}$} \\
\hline & & & & & & & \\
\hline 2" & 50,80 & 0,00 & 0,00 & 0,00 & 0,00 & 0,00 & 0,00 \\
\hline 1" & 25,40 & 0,00 & 17,50 & 0,00 & 0,00 & 0,00 & 0,00 \\
\hline 3/8" & 9,53 & 52,50 & 25,00 & 32,50 & 20,00 & 0,00 & 0,00 \\
\hline 4 & 4,80 & 7,50 & 12,50 & 17,50 & 12,50 & 22,50 & 15,00 \\
\hline 10 & 2,09 & 12,50 & 12,50 & 12,50 & 12,50 & 7,50 & 7,50 \\
\hline 40 & 0,42 & 13,50 & 10,00 & 15,00 & 20,00 & 35,00 & 27,50 \\
\hline 200 & 0,08 & 14,00 & 22,50 & 22,50 & 35,00 & 35,00 & 50,00 \\
\hline Total & - & 100 & 100 & 100 & 100 & 100 & 100 \\
\hline
\end{tabular}

Fonte: Adaptação DNIT (2006).

Na seleção das granulometrias, as especificações técnicas definem intervalos que devem ser obedecidos pelos percentuais passantes em cada peneira. Esse tipo de metodologia é adotado tanto em nível nacional quanto internacionalmente (FERREIRA et al., 2016).

Como procedimento inicial e em conformidade com a norma NBR 6457/86, caracterizou-se o solo proveniente da jazida de cascalho através do uso de ensaios de granulometria conjunta, massa específica dos sólidos e Limites de Liquidez e de Plasticidade, conforme indicado no quadro 1.

QUADRO 1 Ensaios de caracterização do solo.

\begin{tabular}{|c|c|c|c|}
\hline \multicolumn{4}{|c|}{ Amostra Natural } \\
\hline Granulometria & Massa Específica & & Limites de Atterberg \\
\hline Conjunta & dos Sólidos & LL & LP \\
\hline
\end{tabular}

As amostras, em laboratório, foram secas à sombra, destorroadas e então peneiradas segundo a sequência de peneiras indicadas nas faixas granulométricas do DNIT (2006), conforme Tabela 1. Em seguida, foram homogeneizadas, quarteadas e tiveram as suas umidades higroscópicas determinadas.

Em conseguinte, procedeu-se à compactação do material e aos ensaios CBR (Índice de Suporte Califórnia) e RCS (Resistência à Compressão Simples). Estes ensaios foram realizados para as amostras no estado natural, adequadas às faixas granulométricas em estudo, porém sem estabilizante, e quando estabilizadas com $8 \%$ de cal em relação ao peso seco, conforme indicado no quadro 2. 
QUADRO 2. Matriz de ensaios realizados.

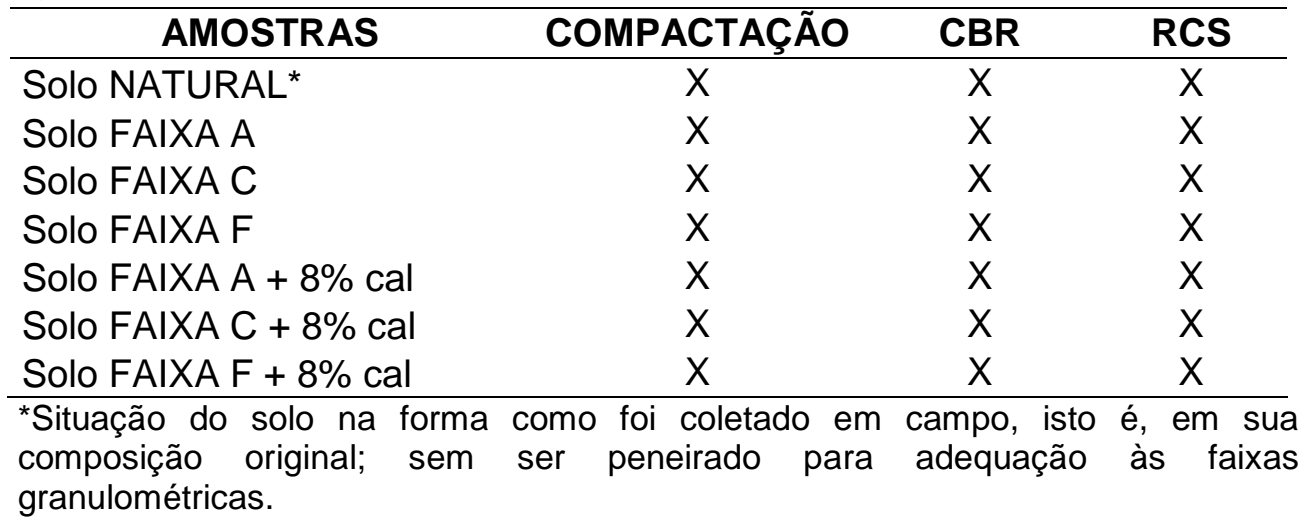

Com base nas normas DNIT - ME 049/94, se é permitido escolher a energia utilizada na compactação segundo os parâmetros de projeto. Dessa forma, para realização do ensaio de compactação foi utilizada a energia intermediária. Para o ensaio de CBR, a energia de compactação empregada foi a intermediária, onde se aplicam 26 golpes por camada em 5 camadas, utilizando-se do soquete padrão de $4,54 \mathrm{~kg}$ e o cilindro Califórnia. A escolha dessa energia baseou-se no suposto tráfego a que se submeteria a amostra e nos materiais estudados. Dessa forma, procedeu-se à confecção dos corpos de prova dos CBRs de 5 pontos, obtendo-se os parâmetros da compactação paralelamente.

Para o cálculo do ensaio CBR são utilizadas as penetrações 2,5 e 5,0 mm; sendo que se adota o maior valor encontrado entre eles.

$\mathrm{O} \mathrm{CBR}_{2,5}$ foi obtido a partir do uso da seguinte Equação (1):

$$
\mathrm{CBR}_{2,5}=(\text { carga } * 100) / 1350
$$

$\mathrm{O} \mathrm{CBR}_{5,0}$ foi obtido a partir do uso da seguinte Equação (2):

$$
\mathrm{CBR}_{5,0}=\left(\text { carga }{ }^{*} 100\right) / 2050
$$

Para o ensaio de RCS, o qual foi realizado de acordo com a norma NBR 12025/90, os corpos de provas foram moldados em umidade ótima e peso específico aparente seco máximo, ambos determinados no ensaio de compactação, utilizandose a energia intermediária. Foram compactados 3 corpos de prova para cada faixa granulométrica e trabalhou-se com a média dos resultados por faixas. Além disso, as bases dos corpos de prova foram niveladas com o uso de enxofre, para melhor ajuste à prensa.

De modo geral, para todos os ensaios, quando o material empregado ao passar pelas peneiras apresentava quantidades de retidos inferiores às indicadas na tabela do DNIT (2006), substituía-se essas quantidades necessárias pelo emprego de brita 1 e de brita 0 .

\section{RESULTADOS E DISCUSSÃO}

O solo oriundo da jazida de cascalho apresentou a seguinte caracterização granulométrica: $13 \%$ de pedregulho; $63 \%$ de areia; $13 \%$ de argila e $11 \%$ de silte. À continuação, apresentou massa específica dos sólidos no valor de $2,664 \mathrm{~g} / \mathrm{cm}^{3}$, 
limite de liquidez de 37,56\% e limite de plasticidade de 23,33\%. A Figura 1 apresenta a análise granulométrica do solo em seu estado natural.

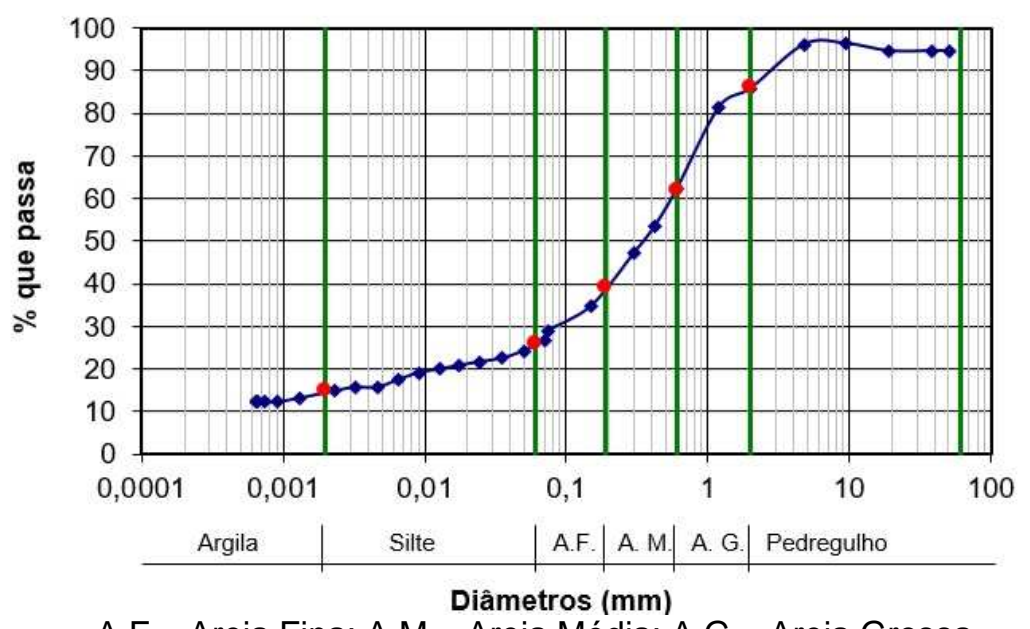

A.F - Areia Fina; A.M - Areia Média; A.G - Areia Grossa

FIGURA 1. Curva granulométrica do solo em estudo.

Após a determinação dos ensaios de caracterização do solo, foi executada a classificação dos materiais em conformidade com a TRB (Transportation Research Board). Com base nesses resultados verificou-se que esse solo de cascalheira é indicado como sendo um material de excelente a bom emprego como subleito. Com relação ao ensaio de CBR para amostras não estabilizadas com cal, obtiveram-se os resultados apresentados na Tabela 2.

TABELA 2. Resultados CBR para amostras sem adição de cal.

\begin{tabular}{cccc}
\hline & Faixa A (\%) & Faixa C (\%) & Faixa F (\%) \\
\hline & 10,89 & 45,19 & 3,63 \\
& 63,41 & 43,85 & 10,4 \\
CBR $_{2,5}$ & 14,37 & 5,48 & 31,85 \\
& 8,3 & 2,67 & 13,7 \\
& 6,67 & 2 & 3,21 \\
\hline \multirow{6}{*}{ CBR $_{5,0}$} & 13,9 & 44,24 & 4,64 \\
& 66,05 & 62,15 & 11,8 \\
& 25,95 & 8,44 & 31,95 \\
& 12,98 & 3,7 & 20,59 \\
& 14 & 2,79 & 4,38 \\
\hline
\end{tabular}

As amostras estabilizadas com cal apresentaram os resultados CBR evidenciados na Tabela 3 a seguir.

\begin{tabular}{|c|c|c|c|c|c|c|c|c|c|c|c|}
\hline \multicolumn{4}{|c|}{ FAIXA A + $8 \%$ CAL } & \multicolumn{4}{|c|}{ FAIXA C + $8 \%$ CAL } & \multicolumn{4}{|c|}{ FAIXAF + $8 \%$ CAL } \\
\hline \multicolumn{2}{|c|}{$\mathrm{CBR}_{2,5}$} & \multicolumn{2}{|c|}{$\mathrm{CBR}_{5,0}$} & \multicolumn{2}{|c|}{$\mathrm{CBR}_{2,5}$} & \multicolumn{2}{|c|}{$\mathrm{CBR}_{5,0}$} & \multicolumn{2}{|c|}{$\mathrm{CBR}_{2,5}$} & \multicolumn{2}{|c|}{$\mathrm{CBR}_{5,0}$} \\
\hline $\begin{array}{c}\text { CARGA } \\
(\mathrm{kg})\end{array}$ & $\mathrm{CBR}_{2,5}$ & $\begin{array}{c}\text { CARGA } \\
(\mathrm{Kg})\end{array}$ & $\mathrm{CBR}_{5,0}$ & CARGA & $\mathrm{CBR}_{2,5}$ & $\begin{array}{c}\text { CARGA } \\
(\mathrm{Kg})\end{array}$ & $\mathrm{CBR}_{5,0}$ & CARGA & $\mathrm{CBR}_{2,5}$ & $\begin{array}{c}\text { CARGA } \\
(\mathrm{Kg})\end{array}$ & $\mathrm{CBR}_{5,0}$ \\
\hline 854 & 63,26 & 1442 & 70,34 & 1255 & 92,96 & 1649 & 80,44 & 958 & 70,96 & 1271 & 62 \\
\hline 1276 & 94,52 & 1725 & 84,15 & 2039 & 151,04 & 2485 & 121,22 & 1871 & 138,59 & 2229 & 108,73 \\
\hline 1489 & 110,3 & 2046 & 90 & 2281 & 168,96 & 3050 & 148,78 & 2149 & 159,19 & 2580 & 125,85 \\
\hline 2596 & 192,3 & 4255 & 207,56 & 2233 & 165,41 & 3797 & 185,22 & 3004 & 222,52 & 3741 & 182,49 \\
\hline 1076 & 79,7 & 2560 & 124,88 & 526 & 38,96 & 1268 & 61,85 & 1029 & 76,22 & 1893 & 92,34 \\
\hline
\end{tabular}

TABELA 3. Resultados CBR para amostras estabilizadas com cal. 
Pode-se observar que o material, atendendo às solicitações das faixas granulométricas $\mathrm{A}, \mathrm{C}$ e $\mathrm{F}$ e, quando combinado com cal no teor de $8 \%$ em relação à sua massa seca, teve um acréscimo em sua resistência para a sequência de faixas estudada. Esse procedimento confirmou a necessidade de se ensaiar o material segundo as especificações para solo-cal, ou seja, empregar o ensaio de RCS por um período de cura de 14 dias. Dessa forma, a Tabela 4 apresenta os resultados obtidos em relação aos ensaios de compactação, de CBR e de RCS para as amostras estabilizadas com cal.

TABELA 4. Resultados dos ensaios de compactação, de CBR e de RCS.

\begin{tabular}{|c|c|c|c|c|c|}
\hline \multirow[b]{2}{*}{ AMOSTRAS } & \multicolumn{2}{|c|}{ COMPACTAÇÃO } & \multicolumn{2}{|c|}{ CBR } & \multirow{2}{*}{$\begin{array}{c}\text { RESISTÊNCIA } \\
\text { À } \\
\text { COMPRESSÃO } \\
\text { SIMPLES } \\
\text { (MPa) }\end{array}$} \\
\hline & $W_{\text {ót (\%) }}$ & $\begin{array}{l}Y_{\text {dmáx }} \\
(\mathrm{g} / \mathrm{cm})\end{array}$ & $\mathrm{CBR}_{(\%)}$ & $\begin{array}{c}\text { Expansão. } \\
\text { CBR (\%) }\end{array}$ & \\
\hline Solo NATURAL & 11,60 & 1,99 & 56,4 & 0,16 & 0,26 \\
\hline Solo FAIXA A & 6,99 & 2,21 & 24 & 0,07 & 0,075 \\
\hline Solo FAIXA C & 8,21 & 2,23 & 8,2 & 0,07 & 0,075 \\
\hline Solo FAIXA F & 11 & 2,02 & 31,8 & 0,96 & 0,170 \\
\hline Solo FAIXA A + $8 \%$ cal & 6,77 & 2,12 & 109,4 & 0,07 & 0,423 \\
\hline Solo FAIXA C + $8 \%$ cal & 7,63 & 2,13 & 169 & 0,07 & 0,410 \\
\hline Solo FAIXA F + 8\% cal & 12,05 & 1,91 & 158,9 & 0,07 & 0,475 \\
\hline
\end{tabular}

Trabalhando-se na energia Proctor intermediária e sem acréscimo de cal, a faixa $F$, a qual contém maior quantidade de finos em sua composição, apresentou maior capacidade de suporte, evidenciando um CBR na ordem de 31,8\%. Já o estudo do material com acréscimo de $8 \%$ de cal, indicou que as faixas $\mathrm{C}$ e $\mathrm{F}$ apresentaram melhores resultados CBR, sendo a faixa $\mathrm{C}$, ainda, a que apresenta valor ligeiramente maior.

Com relação aos ensaios RCS realizados nos corpos de provas estabilizados com cal, os valores encontrados ficaram abaixo daquele sugerido pela norma DNER-ME 180/94 que rege os ensaios solo-cal, ou seja, 2,17 Mpa para 14 dias de cura. Ainda assim, a faixa $F$ foi a que apresentou maior valor de RCS, com resultado superior aos demais.

Tais melhoras proporcionadas pela cal às propriedades físicas e mecânicas do solo já foram evidenciadas em outros propósitos científicos. Segundo EMMERT (2010) a adição de $2 \%$ de cal, com cura de 1, 7 e 28 dias, para um solo classificado como A-4(2), segundo classificação da Transportation Research Board (TRB), implica em ganhos de resistência à compressão simples e índice de suporte Califórnia em comparação ao solo puro, além de aumento desses parâmetros com o tempo de cura.

De acordo com GUÉRIOS (2012), um solo quando acrescido de cal hidratada em diferentes porcentagens, tem suas características melhoradas notavelmente. Segundo o estudo do autor, uma mistura solo-cal com presença de $10 \%$ de cal hidratada resulta em melhoras evidentes ao solo trabalhado, favorecendo todos os aspectos inerentes ao mesmo. O mesmo se infere para dados apresentados por MALANCONI (2013), o qual trabalha aditivos a solos tropicais para utilização como camada de pavimento. Segundo o autor, o uso de $6 \%$ de cal nas análises de CBR e RCS evidenciam grande melhora nessas propriedades do solo, ainda que menores quando comparadas ao uso de cimento. 
Utilizando-se também de cal e cimento para realizar a caracterização geotécnica e melhoramento de determinado solo, COSTA (2015) evidencia o acréscimo na resistência e no suporte do solo proporcionados pela cal. Ainda que, segundo o autor, a utilização do cimento apresente valores mais significativos, o uso de cal se enquadra como um dos melhores resultados obtidos no estudo.

A utilização da cal como estabilizante combinada com outros aditivos também é prática comum no processos de estabilização. Resultados apresentados por KOLAY et al. (2011) mostram que cimento, cal e a combinação desses com cinza volante em um solo argiloso, obtêm significantes melhoras em resistência. Ainda que o cimento apresente melhor resultado, segundo o autor a mistura de cal e cinza volante atinge quase $80 \%$ dos resultados obtidos com uma porcentagem alta de cimento.

Comparativamente aos estudos anteriormente citados, neste trabalho a utilização de $8 \%$ de cal ao solo demostra acréscimo nos índices de suporte e resistência para todas as faixas granulométricas, se comparadas ao solo sem aditivo. Para a mineralogia e as granulometrias estudadas, o acréscimo nos valores de CBR e RCS proporcionados pela cal reforça a ideia geral dos benefícios da utilização da mesma, apresentada pelos autores citados.

As Figuras 2 e 3 ilustram o ensaio CBR e o ensaio de RCS, respectivamente.

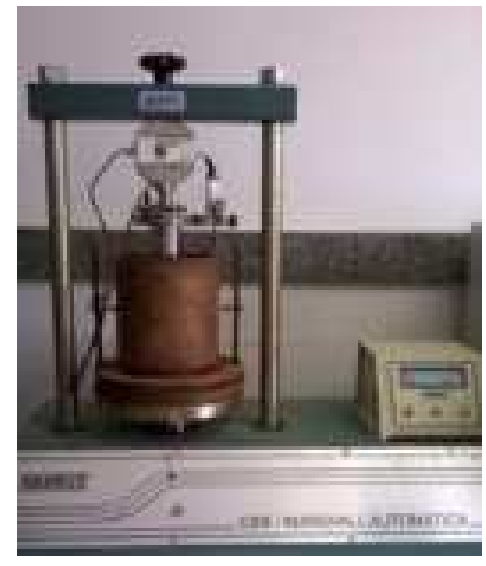

FIGURA 2. Amostra em ensaio CBR.

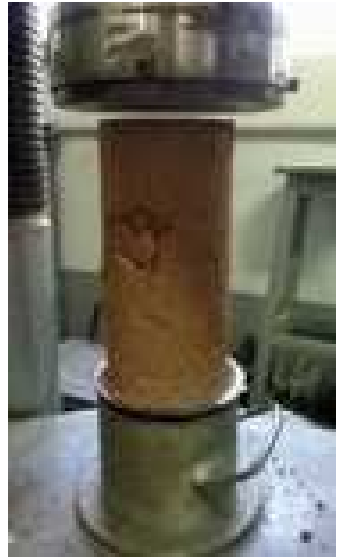

FIGURA 3. Amostra em ensaio RCS.

\section{CONCLUSÃO}

- A classificação da amostra na presença de cal, segundo os resultados CBR expostos, mostra que a mesma é indicada como um material de bom comportamento para ser utilizado como subleito.

- Os materiais tratados com cal apresentam maiores valores de CBR e de RCS nas faixas $C$ e $F$, isto é, naquelas que apresentam maiores quantidades de finos em sua composição. Portanto, as faixas mais consecutivas da norma DNIT (2006) apresentam os melhores resultados de suporte e resistência dentre as faixas analisadas.

- Quanto aos valores de RCS que se apresentaram abaixo do valor estipulado pela norma DNER-ME 180/94, a solução encontra-se na substituição da energia de compactação, de intermediária para modificada, ou no emprego de um teor maior de cal, ou ainda, na combinação da nova energia com o aumento do teor de cal, levando-se em consideração os aspectos técnicos e econômicos. 
- Os índices de suporte e de resistência apresentados pelos materiais tratados com cal indicam que os mesmos, em especial a faixa $\mathrm{C}$ e $\mathrm{F}$, possuem um bom comportamento em relação à estabilidade, implicando também em uma reduzida perda de material frente à precipitação e ao desgaste causado pelo tráfego.

\section{REFERÊNCIAS}

ARAUJO, V. M. C.; BRANCO, V. T. F. C.; LIMA, D. M.; CAVALCANTE, R. M. Avaliação ambiental da utilização de solo contaminado por derivados de petróleo (SCDP) em misturas asfálticas. Revista Transportes, v. 24, n. 2, 2016.

ASSOCIAÇÃO BRASILEIRA DE NORMAS TÉCNICAS - ABNT. DNIT - IPR 719. Manual de Pavimentação. 3ª̣ Edição. Rio de Janeiro, 2006.

. DNIT - ME 049/94. Solos - determinação do Índice de Suporte Califórnia utilizando amostras não trabalhadas. Rio de Janeiro, 1994.

NBR 6457. Amostras de solo - Preparação para ensaios de compactação e ensaios de caracterização. Rio de Janeiro, 1986.

. NBR 12025. Solo-cimento - Ensaio de compressão simples de corpos-de-prova cilíndricos. Rio de Janeiro, 1990.

CNT - CONFEDERAÇÃO NACIONAL DO TRANSPORTE. Anuário CNT de Transportes - Estatísticas Consolidadas 2016 - 2ª tiragem. Brasília: CNT, 2016.

COSTA, L. F. C. Estudo de solo de alteração do jurássico superior: Caracterização geotécnica e melhoramento por cal e cimento. In: Geologia Aplicada - Geologia de Engenharia. Lisboa, 2015.

DEPARTAMENTO NACIONAL DE ESTRADAS DE RODAGEM. DNER-ME 180/94: Solos Estabilizados com Cinza Volante e Cal Hidratada - Determinação da Resistência à Compressão Simples. Rio de Janeiro, 1994.

DICIONÁRIO LIVRE DE GEOCIÊNCIAS. Calcinação. Disponível em: < http://www.dicionario.pro.br/index.php/Calcina\%C3\%A7\%C3\%A3o> Acessado em: 28/06/2016

EMMERT, F. Avaliação do emprego de técnica de estabilização de solo na melhoria de estradas florestais da região de Niquelânicia-GO. In: Ciências Florestais. Universidade de Brasília, Brasília, 2010.

FERREIRA, J. L. S.; SOARES, J. B.; BASTOS, J. B. S. Métodos de seleção granulométrica com foco na resistência à deformação permanente. Revista Transportes, v. 24, n. 2, 2016.

GONDIM, L. M. Estudo experimental de misturas solo-emulsão aplicado às rodovias do Agropólo do baixo Jaguaribe - CE. In: Engenharia de Transportes. Universidade Federal do Ceará, Fortaleza, 2008. 
GUÉRIOS, E. M. Estudo do melhoramento de solo com adição de cal hidratada para seu uso em pavimento urbano. 2012. 69 f. In: Engenharia de Produção Civil. Universidade Tecnológica Federal do Paraná. Curitiba, 2012.

GUYER, J. P. Introduction to soil stabilization in pavements. 2011. $28 f$. Continuing Education and Development, New York. Nova lorque, Estados Unidos, 2011. Notas de aula.

KLINSKY, L. M. G.; BARDINI, V. S. S.; FABBRI, G. T. P. Efeito da adição de areia de fundição residual e cal a solos argilosos no módulo de resiliência. Revista Transportes, v. 22, n. 2, 2014.

KLINSKY, L. M. G.; FABBRI, G. T. P.; FURLAN, A. P. Efeito da adição de areia de fundição e cal em algumas propriedades de solos argilosos. Revista Transportes, v. 20, n. 1, 2012.

KOLAY, P. K.; AMINUR, M. R.; TAIB, S. N. L.; et al, Stabilization of Tropical Peat Soil from Sarawak with Different Stabilizing Agents. Geotechnical and Geological Engineering. v.29, n.6, p.1135-1141, 2011.

LAMBE, T. W.; MICHAELS, A. Altering soil properties with chemicals. Chemical Engineering. n. 6, v. 32, p. 488-492, 1954.

MALANCONI, M. Considerações sobre misturas de solos tropicais estabilizados quimicamente para uso como camada de pavimento urbano. In: Engenharia Urbana. Universidade Federal de São Carlos, 2013.

MINASCAL. Cal Hidratada. Vantagens/Utilidades. Disponível em: < http://www.minascal.com.br/>. Acesso em: 22/06/2016.

MUKESH, H.; PATEL, A. A Review on Effects of Stabilizing Agents for Stabilization of Weak Soil, Journal of Civil and Environmental Research, Vol. 2, No. 6. 2012.

OLIVEIRA, H. M. Aglomerantes. In: BAUER, L. A. Falcão. (Coord.). Materiais de Construção. Rio de Janeiro: LTC, 2011. 1v. p. 11-34.

OLIVEIRA, T. M.; SANT'ANNA, G. I.; MACHADO, C. C. Efeito da adição da cal na estabilidade e na resistência à compressão da mistura solo-grits. Rem: Revista Escola de Minas. v. 62, n. 1, p. 87-92, 2009.

SILVA, D. P. Estudo do comportamento geológico de solos estabilizados com cimento: processos gerais de estabilização. Lisboa: Ministério de obras públicas. Laboratório Nacional de Engenharia Civil, 343p. 1968.

SNV - SISTEMA NACIONAL DE VIAÇÃO. SNV 2016 - Atualizado até 16/09/2016: SNV 2016 COMPLETO - Rede rodoviária sob jurisdição do Ministério dos Transportes. Disponível em: < http://www.dnit.gov.br/sistema-nacional-deviacao/sistema-nacional-de-viacao > Acesso em: 21/09/2016 
SOUSA, A. T. Estudo de parâmetros de dois tipos de solos característicos do Distrito Federal estabilizados com cal. 2013. 70 f. in: Engenharia Civil - Centro Universitário de Brasília - UniCEUB, Brasília, 2013.

VIZCARRA, G. O. C. Aplicabilidade de Cinzas de Resíduo Sólido Urbano Para Base de Pavimentos. In: Engenharia Civil. PUC-Rio, Rio de Janeiro, 2010. 